\title{
USO DE MEDICAMENTOS DURANTE A LACTAÇÃO: UM FATOR PARA SUSPENSÃO DO ALEITAMENTO MATERNO ${ }^{1}$
}

\author{
Jefferson Marlon de Medeiros Pereira Maciel, Universidade Federal de Campina Grande \\ (UFCG), jeffersonmmpmaciel@ hotmail.com \\ Andreza Guedes Barbosa Ramos, Universidade Federal de Campina Grande (UFCG), \\ andrezaurca@gmail.com
}

\begin{abstract}
RESUMO
A amamentação tem sua importância abordada sob um ponto de vista multiprofissional, envolvendo aspectos imunológicos, nutricionais, cognitivos, psicoafetivos, econômicos e sociais. Não obstante, diversos fatores podem levar à suspensão precoce do aleitamento materno, dentre os quais destacamse os problemas relacionados aos riscos de exposição das lactentes a medicações maternas. $\mathrm{O}$ estudo trata-se de uma revisão e atualização da literatura disponível sobre o uso de medicamentos durante o período de amamentação, visando contribuir com informações úteis para profissionais de saúde na assistência materno-infantil. Foram selecionados artigos nos bancos de dados eletrônicos Pubmed, Medline, Lilacs e SciELO publicados nos últimos vinte anos nas línguas portuguesa e inglesa, utilizando os descritores "aleitamento materno", "lactação", e "uso de medicamentos". Os estudos encontrados mostraram o uso frequente de medicamentos pela mulher durante a amamentação, a sua grande influência no desmame precoce indevido, e ainda um preocupante desconhecimento dos profissionais de saúde sobre o tema. É pertinente aos profissionais de saúde uma reflexão e revisão das práticas adotadas, propiciando um tratamento adequado à nutriz, contribuindo para a manutenção do aleitamento materno. Faz-se necessário ainda, maior investimento em pesquisas que determinem o grau de segurança dos fármacos durante a lactação.
\end{abstract}

PALAVRAS-CHAVE: aleitamento materno; uso de medicamentos; desmame.

\section{USE OF MEDICATIONS DURING LACTATION: A FACTOR OF SUSPENSION OF BREASTFEEDING}

\begin{abstract}
Breastfeeding has its importance addressed from a multiprofessional point of view, involving immunological, nutritional, cognitive, psychoaffective, economic and social aspects. Nevertheless, several factors can lead to early discontinuation of breastfeeding, among which are the problems related to the risks of exposure of infants to maternal medications. The study is a review and update of the available literature on the use of medicines during the breastfeeding period, aiming to contribute information useful for health professionals in maternal and child care. We selected articles in the

${ }^{1} \mathrm{O}$ presente trabalho não contou com apoio financeiro de nenhuma natureza para sua realização.
\end{abstract}


electronic databases Pubmed, Medline, Lilacs and SciELO published in the Portuguese and English languages, using the descriptors "breastfeeding", "lactation" and "use of drugs". The studies found showed the woman's frequent use of medications during breastfeeding, her great influence on undue early weaning, and a worrying lack of knowledge of health professionals about the subject. It is up to the health professionals to reflect and review the practices adopted, providing an adequate treatment to the nursing woman, besides contributing to the maintenance of breastfeeding. It is necessary to invest more in research that determines the degree of safety of the drugs during lactation.

KEYWORDS: breastfeeding; use of medicines; weaning.

\title{
USO DE MEDICAMENTOS DURANTE LA LACTACIÓN: UN FACTOR DE LA SUSPENSIÓN DEL ALCANCE MATERNO
}

\begin{abstract}
RESUMEN
La lactancia materna tiene su importancia abordada desde un punto de vista multiprofesional, involucrando aspectos inmunológicos, nutricionales, cognitivos, psicoactivos, económicos y sociales. No obstante, diversos factores pueden llevar a la suspensión temprana de la lactancia materna, entre los que se encuentran los problemas relacionados con los riesgos de exposición de los lactantes a medicamentos maternos. El estudio se trata de una revisión y actualización de la literatura disponible sobre el uso de medicamentos durante el período de lactancia, con el objetivo de contribuir con informaciones útiles para profesionales de salud en la asistencia materno-infantil. Se seleccionaron artículos en los bancos de datos electrónicos Pubmed, Medline, Lilacs y SciELO publicados en los últimos veinte años, en las lenguas portuguesa e inglesa, utilizando los descriptores "lactancia materna", "lactancia", y "uso de medicamentos". Los estudios encontrados mostraron el uso frecuente de medicamentos por la mujer durante la lactancia, su gran influencia en el destete precoz indebido, y aún un preocupante desconocimiento de los profesionales de salud sobre el tema. A los profesionales de la salud una reflexión y revisión de las prácticas adoptadas, propiciando un tratamiento adecuado a la nutrición, además de contribuir al mantenimiento de la lactancia materna. Se requiere una mayor inversión en investigaciones que determinen el grado de seguridad de los fármacos durante la lactancia.
\end{abstract}

PALABRAS CLAVES: lactancia materna; uso de medicamentos; desmame.

\section{INTRODUÇÃO}

A amamentação natural e a sua importância tem sido abordadas sob um ponto de vista multiprofissional, envolvendo aspectos imunológicos, nutricionais, cognitivos, psicoafetivos, econômicos e sociais (NEIVA et al., 2003, p. 07), sendo decorrente de uma série de impulsos biológicos, instintivos e comportamentais, estes apresentados por todos os recém-nascidos de mamíferos (MARQUES; COTTA; PRIORE, 2011, p. 2462).

$\mathrm{O}$ ato da amamentação propicia o contato físico entre mãe e filho, estimulando os sentidos e trazendo conforto para o recém-nascido, estabelecendo o vínculo entre mãe e o neonato. Os aspectos psicológicos do aleitamento materno 
estão relacionados ao desenvolvimento da personalidade do indivíduo quando adulto (TEDESCHI, 1982, p. 145).

A alimentação exclusiva com leite materno logo após o nascimento é reconhecidamente a melhor forma de proteger o recém-nascido das enfermidades infecciosas (BALMER; WHARTON, 1989, p. 1673), e por isso é recomendada pela Organização Mundial da Saúde até os seis meses de idade, passando a ser acompanhada com outros alimentos até os dois anos ou mais. Através do colostro e do leite humano, a mãe se torna a principal fonte de microorganismos importantes (imunidade passiva) para o estabelecimento da microbiota digestiva do recém-nascido, tanto no parto quanto na amamentação (DE ALMEIDA; NOVAK, 2004, p. 121). O colostro humano é definido como o primeiro produto da secreção láctea da nutriz até o sétimo dia de pós-parto e de extrema importância para a hidratação do bebê.

Não obstante, apesar de todas essas vantagens, diversos fatores podem levar à suspensão precoce do aleitamento materno, dentre os quais encontram-se os problemas relacionados aos riscos de exposição dos lactentes a medicações maternas (HOWARD; LAWRENCE, 1999, p. 453).

Após o parto, o uso de medicamentos é frequente entre as lactantes, devido à necessidade de tratamento para combater infecções, depressão, além das doenças crônicas que requerem medicamentos de uso contínuo (SCHIRM; SCHWAGERMANN; TOBI H, 2004, p. 387). Nesse cenário, muitas informações e referências na literatura sobre a relação de drogas e aleitamento materno estão disponíveis, porém muitos profissionais de saúde, especialmente médicos e enfermeiros, por motivos diversos, preferem interromper a amamentação em vez de se esforçarem para compatibilizá-la com a terapêutica materna.

Em geral, os profissionais de saúde que atuam prescrevendo medicamentos para lactantes devem se basear na relação risco/benefício e conhecer as categorias de risco das drogas, que vão desde as mais seguras até as contraindicadas. A amamentação somente deverá ser interrompida se existirem evidências de que a droga usada pela lactante é nociva para o lactente, ou quando não houver informações a respeito e a droga não puder ser substituída por outra que seja compatível com a amamentação (CHAVES et al., 2007, p. 287).

A associação entre uso de medicamentos pela nutriz e desmame reforça a importância da ciência do tema. Além disso, face à dinâmica do conhecimento e do surgimento de novas drogas, as informações necessitam ser constantemente atualizadas para proporcionar maior segurança para ao clínico responsável pela assistência à mulher lactante. Assim, este trabalho 
é uma revisão e uma atualização da literatura disponível sobre o uso de medicamentos durante o período de amamentação, visando contribuir com informações úteis para profissionais de saúde na assistência materno-infantil.

\section{MÉTODOS}

Este trabalho consiste de uma revisão da literatura abrangendo estudos mais relevantes sobre o uso de medicamentos durante a lactação. Foram selecionados artigos nos bancos de dados eletrônicos Pubmed, Medline, Lilacs e SciELO publicados nos últimos vinte anos, nas línguas portuguesa e inglesa, utilizando os descritores "aleitamento materno", "lactação", e "uso de medicamentos". Também foram utilizados livros, textos recentes e diretrizes considerados relevantes para a realização dessa revisão.

Para a discussão dos dados, foram abordados aspectos farmacológicos determinantes na escolha dos fármacos e ressaltados os medicamentos mais seguros e os contraindicados, além de cuidados especiais com alguns medicamentos.

\section{RESULTADOS E DISCUSSÃO}

O uso de medicamentos pela mulher durante a amamentação é uma prática muito frequente, como mostrado em vários estudos em diferentes fases da lactação (CHAVES et al., 2007, p. 285). No período intraparto, um estudo multicêntrico demonstrou uso de medicamentos por quase $80 \%$ das mulheres (DA FONSECA; DA FONSECA; BERGSTENMENDES, 2002, p. 209). Em maternidades de Belo Horizonte, Minas Gerais, estudo realizado com 2.173 parturientes mostrou que 96,2\% delas receberam prescrição medicamentosa no pós-parto imediato: $98,3 \%$ eram drogas sem contraindicação, $16,2 \%$ não tinham informações seguras e $0,14 \%$ eram contraindicadas à amamentação. A análise por meio dos grupos farmacológicos mostrou que a maior parte das mulheres estava fazendo uso de analgésicos, antiinflamatórios não esteroidais e antibióticos (LAMOUNIER; 2002, p. 59). Em Itaúna, município próximo à capital mineira, $100 \%$ das puérperas receberam medicamentos no pós-parto imediato, com média de 4,3 fármacos por mulher. Após a alta hospitalar, 98\% das mulheres relataram o emprego de pelo menos um fármaco (CHAVES, 2004, p. 113). 
Sobre as drogas para as quais recomenda-se uso criterioso durante a amamentação, verificou-se uma porcentagem pequena de prescrições, o que pode ser explicado pelo receio de se prescrever drogas potencialmente tóxicas para as lactentes ou com possíveis efeitos deletérios já descritos. Quanto aos grupos farmacológicos mais prescritos, não há nenhuma surpresa, por serem essas as drogas utilizadas para aliviar os sintomas mais comuns no período pós-parto.

A frequente necessidade de medicamentos na lactação deve ser encarada com preocupação devido à conhecida relação entre tal uso e o desmame injustificado. Ito et al. (2000, p. 121) demonstraram que mulheres em tratamento farmacológico de doenças crônicas iniciavam a amamentação menos frequentemente do que as mulheres da população geral e, quando iniciavam, desmamavam seus filhos mais precocemente. Em Montes Claros, Minas Gerais, a análise de 50 mulheres com interrupção do aleitamento demonstrou que, em $20 \%$ delas o uso de medicamentos foi o motivador (CALDEIRA; GOULART, 2000, p. 68).

A interrupção da amamentação durante o uso de medicamentos só deveria se justificar quando o fármaco em questão fosse contraindicado neste período. Porém, o desconhecimento dos profissionais de saúde sobre o tema, as informações não científicas em bulas de medicamentos, a escassez de informações na literatura sobre a segurança dos fármacos na lactação e o receio materno de usar medicamentos neste período, na literatura são pretextos utilizados para o desmame precoce. Frequentemente, médicos aconselham lactantes em uso de medicamentos a suspender a amamentação, sem avaliar as possíveis consequências não só para o lactente, mas também para o suprimento lácteo materno (HALE, 2004, p. 165). Estudo realizado numa Unidade Básica de Saúde em Fortaleza, Ceará, dentre as 132 mães investigadas, 76 receberam indicação do uso de medicamentos por profissionais da saúde, e 25 se automedicaram. Em contra partida, a maioria das mães, 58\%, não recebeu nenhuma orientação sobre o uso de medicação durante a lactação. Nesse sentido, enfatiza-se, a importância de as mulheres serem orientadas sobre o uso de medicações e aleitamento materno nas consultas de pré-natal e na puericultura, oportunidade para que se busque por informações adequadas e esclareçam dúvidas (MOTA, et al., 2013, p. 143).

No intuito de orientar os profissionais de saúde sobre o uso de medicamentos na lactação, a Academia Americana de Pediatria (AAP) (PEDIATRICS, 2001, p. 779), na última revisão do seu consenso sobre a transferência de drogas para o leite humano, classifica-as em: 
drogas citotóxicas, que podem interferir no metabolismo celular do lactente; drogas de abuso com efeitos adversos descritos no lactente; compostos radioativos que requerem a suspensão temporária da amamentação; drogas com efeitos desconhecidos, mas que requerem preocupação; drogas com efeitos significativos em alguns lactentes e que devem ser usadas com cautela; e drogas compatíveis com a amamentação.

Para que o profissional de saúde possa se orientar sobre o uso de medicamentos na lactação, é imprescindível o conhecimento dos fatores que determinam a segurança para uso nesse período. Tais fatores podem estar relacionados com os aspectos metabólicos e fisiológicos do leite humano, com a mulher, com o lactente ou com o fármaco. (CHAVES; LAMOUNIER, 2004, p. 195).

A composição do leite materno varia conforme a fase da lactação (colostro ou leite maduro) ou até mesmo durante uma mamada (leite anterior ou leite posterior). Tais alterações influenciam na extensão da transferência de fármacos do plasma para o leite, onde se sabe que a transferência é mais facilitada durante os primeiros dias de lactação (BEGG, et al., 2002, p. 326). Contudo, o volume do colostro ingerido nos primeiros dias de vida do bebê é considerado baixo, sendo a dose absoluta de medicamento passada para o recém-nascido durante esse período muito baixa (HALE, et al., 2016, p. 352).

Fatores que reduzem a capacidade da mãe de metabolizar ou excretar a droga podem aumentar a exposição do lactente (CHAVES et al., 2007, p.277). Assim, cuidado especial deve ser tomado ao se prescrever drogas para nutrizes com doenças hepáticas ou renais, pelos níveis mais elevados e pelo maior tempo da droga na circulação materna. A via pela qual a droga é administrada à mãe também tem importância pelos níveis alcançados no plasma materno e, posteriormente, no leite humano. Assim, muitas drogas administradas topicamente ou inaladas não atingem níveis plasmáticos significativos, possuindo níveis lácteos não mensuráveis.

A idade do lactente tem sido apontada como uma das mais importantes variáveis a ser considerada no momento de determinar a segurança do fármaco para uso durante a lactação. É consenso haver um número mais elevado de reações em neonatos e menores de dois meses, e menores são as chances de reações ao avançar da idade, devido ao aprimoramento na maturidade metabólica hepática e na barreira hematoencefálica, bem como, na gradual 
redução da ingesta láctea devido à alimentação complementar (CHAVES; LAMOUNIER, 2004, p. 191).

Conhecer certas características das drogas e sua difusão pelo corpo pode ser útil na identificação do risco de seu uso durante a amamentação. A farmacocinética das drogas varia com alguns dos constituintes do leite e com fatores maternos. Podem influenciar na farmacocinética das drogas fatores como o grau de ionização, a lipossolubilidade, a ligação com proteínas do plasma, o peso molecular, a meia vida de eliminação e a biodisponibilidade (CHAVES et al., 2007, p.283).

Diante desse cenário, o princípio fundamental da prescrição de medicamentos para mães lactantes baseia-se, sobretudo, no binômio risco/benefício (CHAVES; LAMOUNIER, 2004, p. 197). Na tomada de decisões pelo profissional de saúde quanto ao manejo das lactantes em uso de medicamentos, os principais aspectos a serem avaliados incluem os benefícios da amamentação, o impacto dos sintomas e da doença sobre a saúde materna, além do desejo materno. A indicação criteriosa do tratamento materno e a seleção cuidadosa dos medicamentos geralmente permitem que a amamentação continue sem interrupção e com segurança (LAMOUNIER, et al., 2000, p. 105).

\section{CONSIDERAÇÕES FINAIS}

As vantagens e a importância do aleitamento materno são bem conhecidas. Assim, a amamentação somente deverá ser interrompida diante de evidência substancial de que o fármaco usado pela nutriz é nocivo para o lactente.

A associação entre uso de medicamentos pela nutriz e a insuficiente orientação médica ou a não compreensão pelas mães revela a necessidade de constante atualização dos profissionais que transcrevem ou orientam as nutrizes sobre o uso dos fármacos durante a lactação. Durante a consulta, é extremamente importante que a mulher seja assistida em sua totalidade, no esclarecimento de dúvidas e na superação de dificuldades, no papel de mãe e provedora do aleitamento do filho, sendo papel dos profissionais de saúde que atuam nos serviços de saúde prestar atendimento de qualidade, tornando o ato de amamentar prazeroso e não obrigação.

No Brasil, são comercializados cerca de 1.500 princípios ativos, sendo que a grande maioria carece de estudos no que diz respeito à sua transferência para o leite materno e seu 
uso durante a lactação. A carência de informações sobre a segurança para uso na lactação de grande parte dos fármacos dificulta a tomada de decisão no momento da prescrição ou orientação pelo profissional de saúde. Faz-se necessário maior investimento em pesquisas que determinem o grau de segurança dos fármacos durante a lactação. O conhecimento farmacológico permite ao profissional propiciar um tratamento adequado à nutriz, além de contribuir para a manutenção do aleitamento materno.

Cabe, aos profissionais de saúde inseridos numa equipe multidisciplinar, uma reflexão e revisão das práticas adotadas, visando ao emprego de medicamentos estritamente essenciais, com inegáveis benefícios tanto para mulher como para o recém-nascido, bem como pela redução de custos na assistência médica. Ainda é ação do médico, preferir drogas comprovadamente seguras, se possível de administração tópica, ajustando a administração da droga para que não coincida o seu pico plasmático com o horário da amamentação (prefere-se o uso antes ou logo após a amamentação), orientar as mães dos possíveis efeitos colaterais no lactente, e no caso de interrupção temporária da amamentação, o leite materno deve ser retirado com antecedência e estocado em congelador para alimentar o bebê.

\section{REFERÊNCIAS BIBLIOGRÁFICAS}

BALMER, S.; WHARTON, B. Diet and faecal flora in the newborn: breast milk and infant formula. Archives of Disease in Childhood, Londres, v. 64, n. 12, p 1672-1677, dezembro 1989.

BEGG, E. J. et al. Studying drugs in human milk: Time to unify the approach. J Hum Lact, New Zealand, v. 18, n. 04, p. 323-332, novembro 2002.

CALDEIRA, A. P.; GOULART, E. M. A. A situação do aleitamento materno em Montes Claros, Minas Gerais: estudo de uma amostra representativa. J Pediatr (Rio J), Montes Claros, v. 7, n. 6, p. 65-72, fevereiro 2000.

CHAVES, R. G.; LAMOUNIER, J. A. Uso de medicamentos durante a lactação. J. Pediatr. (Rio J.), Porto Alegre, v. 80, n.5, supl. p. s189-s198, novembro 2004.

CHAVES, R. G.; LAMOUNIER, J. A.; CÉSAR, C.C. Medicamentos e amamentação: atualização e revisão aplicadas à clínica materno-infantil. Rev Paul Pediatr, São Paulo, v. 25, n. 03, p276-288, fevereiro 2007. 
CHAVES, R.G. Situação do aleitamento materno e do uso de medicamentos pela nutriz no primeiro ano de vida da criança em Itaúna, MG. 2004. Dissertação (Mestrado). UFMG, Belo Horizonte, 2004.

DA FONSECA, M. R. C. C.; DA FONSECA, E.; BERGSTEN-MENDES, G. Prevalência do uso de medicamentos na gravidez: uma abordagem farmacoepidemiológica. Rev. Saúde Pública, São Paulo, v.36, n.2, p.205-212, abril 2002.

DE ALMEIDA, J. A. G.; NOVAK, F. R. Amamentação: um híbrido natureza-cultura. $J$. Pediatr. (Rio J.), Porto Alegre, v. 80, n. 5, supl. p. s119-s125, novembro 2004.

HALE, T. W. Drug therapy and breastfeeding: pharmacokinetics, risk factors, and effects on milk production. Neoreviews, Elk Grove Village, v. 5, n. 1, p. 164-172, abril 2004.

HALE, T. W. et al. Medications and Mothers' Milk 2017. Nova Iorque: Springer Publishing Company, 2016.

HOWARD, C. R.; LAWRENCE, R. A. Drugs and breastfeeding. Clin Perinatol, Philadelphia, v. 26, n. 2, p. 447-478, abril 1999.

ITO, S. et al. Drug therapy for breastfeeding women. $N$ Engl J Med, Massachusetts, v. 34, n. 3, p. 118-126, julho 2000.

LAMOUNIER, J. A. et al. Medicamentos e amamentação. Revista Médica de Minas Gerais, Belo Horizonte, v. 10, n. 02, p. 101-111, agosto 2000.

LAMOUNIER, J. A. et al. O uso de medicamentos em puérpuras interfere nas recomendações quanto ao aleitamento materno?. J. Pediatr. (Rio J.), Porto Alegre, v.78, n.1, p.57-61, fevereiro 2002.

MARQUES, E. S.; COTTA, R. M. M.; PRIORE, S. E. Mitos e crenças sobre o aleitamento materno. Ciênc. saúde coletiva, Rio de Janeiro, v.16, n.5, p. 2461-2468, maio 2011.

MOTA, L. S. et al. Uso de medicamentos durante a lactação por usuárias de uma unidade básica de saúde. Rev Rene, Fortaleza, v. 14, n. 01, p. 139-147, fevereiro 2013.

NEIVA, F. C. B. et al. Desmame precoce: implicações para o desenvolvimento motor-oral. $J$. Pediatr. (Rio J.), Porto Alegre, v. 79, n. 1, p. 7-12, fevereiro 2003.

PEDIATRICS. American Academy of Pediatrics Committee on drugs. The transfer of drugs and other chemicals into human milk. Pediatrics, Nova Iorque, v. 10, n. 08, p 776-789, setembro 2001. 
SCHIRM, E.; SCHWAGERMANN, M.; TOBI H, J.; Drug use during breastfeeding: a survey from the Netherlands. Eur J Clin Nutr, Londres, v. 58, n. 02, p 386-390, fevereiro 2004.

TEDESCHI, N. Aspectos psicologicos do aleitamento materno. Pediatr. Mod, São Paulo, v. 17, n. 3, p 145-146, agosto 1982. 